\title{
Visual Perception in Autism Spectrum Disorder: A Review of Neuroimaging Studies
}

\author{
Seungwon Chung ${ }^{1}$ and Jung-Woo Son ${ }^{2}$ \\ 1 Department of Psychiatry, Chungbuk National University Hospital, Cheongju, Korea \\ ${ }^{2}$ Department of Neuropsychiatry, College of Medicine, Chungbuk National University, Cheongju, Korea
}

\begin{abstract}
Although autism spectrum disorder (ASD) is a neurodevelopmental disorder characterized by social impairments, patients with ASD frequently manifest atypical sensory behaviors. Recently, atypical sensory perception in ASD has received much attention, yet little is known about its cause or neurobiology. Herein, we review the findings from neuroimaging studies related to visual perception in ASD. Specifically, we examined the neural underpinnings of visual detection, motion perception, and face processing in ASD. Results from neuroimaging studies indicate that atypical visual perception in ASD may be influenced by attention or higher order cognitive mechanisms, and atypical face perception may be affected by disrupted social brain network. However, there is considerable evidence for atypical early visual processing in ASD. It is likely that visual perceptual abnormalities are independent of deficits of social functions or cognition. Importantly, atypical visual perception in ASD may enhance difficulties in dealing with complex and subtle social stimuli, or improve outstanding abilities in certain fields in individuals with Savant syndrome. Thus, future research is required to elucidate the characteristics and neurobiology of autistic visual perception to effectively apply these findings in the interventions of ASD.
\end{abstract}

Key Words: Autism spectrum disorder; Visual perception; Neuroimaging; Functional magnetic resonance imaging.

Received: April 24, 2020 / Revision: May 31, 2020 / Accepted: June 5, 2020

Address for correspondence: Jung-Woo Son, Department of Neuropsychiatry, College of Medicine, Chungbuk National University, 1 Chungdae-ro, Seowon-gu, Cheongju 28644, Korea

Tel: +82-43-269-6187, Fax: +82-43-267-7951, E-mail: mammosss@hanmail.net

\section{INTRODUCTION}

Autism spectrum disorder (ASD) is a neurodevelopmental disorder characterized by deficits in social communication and interaction and by restricted or repetitive patterns of behavior, interests, and activities (RRB) [1]. Many researchers have focused on the social deficit of ASD. Although there is much to be learned regarding its neural underpinnings, involvement of atypical social brain networks is widely acknowledged as a common concept. Social brain networks are supposed to be involved in understanding other's intentions, beliefs, and mental states, such as wishes [2]. A number of neuroimaging studies have suggested altered social brain networks in ASD [3-6].

Individuals with ASD often exhibit atypical sensory behaviors across multiple sensory modalities: indifference to pain, adverse response to specific sounds or textures, excessive smelling or touching of objects, and visual fascination with lights or movement [1]. Sensory abnormality, which has received less attention than social impairment, is a common symptom ob-

This is an Open Access article distributed under the terms of the Creative Commons Attribution Non-Commercial License (https://creativecommons.org/licenses/by-nc/4.0) which permits unrestricted non-commercial use, distribution, and reproduction in any medium, provided the original work is properly cited. served in approximately 95\% of children with ASD [7]. Recently, an increasing number of researchers consider sensory abnormality as a critical symptom. The diagnostic criteria for ASD in the Diagnostic and Statistical Manual of Mental Disorders, Fifth Edition (DSM-5) also included "hyper- or hypo-reactivity to sensory input or unusual interest in sensory aspects of the environment" as an example of RRB [1]. Interestingly, sensory abnormality and social impairment seem to be related to each other in ASD. In infants with ASD, atypical sensory development preceded social communicative symptoms [8], sensory hyporesponsiveness predicted subsequent lower levels of joint attention and language development [9], and altered visual perception predicted diagnostic status of ASD [10]. Thus, sensory impairment may be causal to social impairment in ASD, or the two may interact closely [11,12].

As up to $80 \%$ of the information that the human brain receives from external environment is processed visually [13], vision is regarded as the most important sensory modality. Gaze processing, which is an important prerequisite for joint attention [14], or imitation, which is closely related to empathy [15], do not develop normally without intact visual processing. The infants at risk for autism, who are later diagnosed with ASD, more frequently show normal social behaviors (e.g., 
attending to faces) than typically developing (TD) infants up to 12 months of age [16]. However, they exhibit atypical visual behaviors after 12 months of age, followed by abnormal social behaviors [17]. This observation indicates that defective visual processing may contribute to the occurrence of social impairment. For example, in individuals with ASD, atypical visual perception can complicate the processing of visually presented social cues, and this may eventually result in isolation from confusing social information [18].

Among other sensory domains, altered visual perception has been widely reported in individuals with ASD. For example, individuals with ASD seem to be more biased toward local details than global perception, a phenomenon also known as "looking at the trees, but not at the forest" [11]. Individuals with ASD often show superior visual detection [19], and enhanced visual search may be considered as an early marker of ASD [10]. For some individuals with ASD, their bias toward detail seems to play an important role in developing tremendous Savant skills, such as extremely outstanding visual memory or 3-D drawing performance [20]. In particular, atypical visual perception may affect artistic abilities. In line with this view, characteristics of visual perception in ASD were examined from the perspective of neuroaesthetics [21], which deals with the neurobiological aspects of esthetic experiences [22]. In contrast, other findings suggest that the visual search ability of individuals with ASD does not differ from that of TD individuals [23]. Some researchers argue that local visual perception abilities in individuals with ASD depend on the complexity of stimuli [24] or spatial frequency [25]. Several studies have investigated global motion perception in ASD. Perceiving a single moving object is defined as local motion perception, whereas perceiving multiple objects moving relative to one another is defined as global motion perception [26]. Numerous studies have reported that individuals with ASD show global motion processing deficit $[27,28]$, while other studies have found no difference between ASD and TD individuals [29]. Difficulties in biological motion perception, especially in extracting complex information from the motion, have also been reported [30]. Since human faces are a typical social stimulus, numerous studies have investigated atypical face processing in ASD, which is characterized by social impairment. Individuals with ASD show difficulties in facial recognition [31] and gender discrimination [32], and abnormalities in processing facial emotional expression [33] and eye gaze [34]. The results from studies are inconsistent because familiarity or movement of the face stimuli and subject gaze or attention affects the results [35].

Neuroimaging provides neurobiological insight into various mental states such as emotion, cognition, and behavior. Behavioral impairments may be monitored with neuroimag- ing. Despite normal behaviors, the neurocircuitries related to certain behaviors may exhibit altered activity, or the brain regions, other than those typically involved with behaviors, may be activated. Presently, there is no consensus in the findings of neuroimaging studies examining visual processing in ASD. While some studies have reported abnormalities in early visual processing, others have reported atypical higher order visual processing. Several researchers have argued that it is not the visual processing but altered cognition or attention that is compromised in ASD.

This review examines neuroimaging studies focusing on visual detection, motion perception, and face processing to elucidate the features of visual perception in ASD. We also examined whether these characteristics of visual perception are related to social deficit in ASD.

\section{VISUAL DETECTION}

Individuals with ASD show impairments in multiple cognitive domains such as social interaction and communication, language, and executive functions. However, there is one exception: the ability to find local features in complex visual stimuli, namely visual detection. For successful visual detection, it is necessary to focus on local single details and suppress an urge to perceive global stimuli [36]. Over the past few decades, many investigations have reported superior visual detection in ASD [19,37].

\section{Common findings from neuroimaging studies}

Embedded figures task (EFT) is a popular method that has been used to investigate visual search superiority in ASD, and has been used frequently in functional neuroimaging studies. In this task, a participant is instructed to find a simple target shape hidden within a complex figure [38]. Block design task (BDT) is also a useful evaluation tool, which has originally been used to measure visuospatial ability in the Wechsler Adult Intelligence Scales-Revised (WAIS-R) [39]. In this task, a participant is required to re-create a pattern shown in a picture with all white, all red, or half white and half red blocks in hand. When performing in the scanner, a participant is instructed to count the number of particular shapes in a pattern [36], or to choose a shape that matches another shape at a particular position in a pattern [40]. In the visual search paradigm task developed by Keehn et al. [41], the upright letter "T" is a target, and Ts rotated at $90^{\circ}, 180^{\circ}$, and $270^{\circ}$ are distractors.

Consistent findings from functional magnetic resonance imaging (fMRI) studies that used a visual detection task showed that individuals with ASD, compared to TD individuals, activated posterior brain regions such as the primary visual cortex (V1) and extrastriate cortex more extensively, whereas they 
showed less activity in the frontal regions. In the first fMRI study using EFT by Ring et al. [42], the ventral occipitotemporal regions were activated more in the ASD than in the TD group. These primary and associated visual areas are responsible for early visual processing and are thought to be involved in visual imagery of objects [43]. Individuals with ASD may use a strategy to utilize mental imagery to compare a target shape with complex design [42]. Similarly, in an fMRI study using BDT, among V1, V2, V3, V4, V5, and VP, only V2 showed BDT-specific atypical hemodynamic response in the ASD group [36]. In addition, several studies have reported hyperactivation of occipital regions in ASD individuals during visual detection tasks $[40,44,45]$. Perhaps this atypical brain activity makes features of individual stimuli more prominent, thereby enhancing local visual processing [44]. These results show that visual perception in ASD is atypical in early stage.

In contrast to individuals with ASD, TD individuals seem to involve higher order cognitive processing while performing visual detection tasks. In an fMRI study with adult participants, TD participants showed stronger activity in the right dorsolateral prefrontal cortex (DLPFC) [42]; in other studies involving children, TD participants showed greater activity in the left DLPFC compared to participants with ASD [46]. This indicates that TD individuals utilize both spatial working memory (right DLPFC) and verbal working memory (left DLPFC) as strategies for visual detection [47]. Relative activation of the medial prefrontal cortex was also observed in TD individuals $[40,45,46]$. It is possible that TD individuals perform global processing to reveal overall meaning and coherence of figures during visual detection tasks [40]. In contrast to these findings, it was reported that individuals with ASD overactivated the fronto-parietal region during visual detection [41] or underactivated the posterior brain regions during visual detection compared to TD individuals [48]. One report showed no differences between the ASD and TD groups [49].

\section{Visual detection and conceptual models of ASD}

The characteristics of visual processing in ASD as described above are partially consistent with the weak central coherence hypothesis. Central coherence is the ability to integrate information into one meaningful whole. According to the weak central coherence hypothesis, individuals with ASD exhibit a defect in the higher order mechanism of integrating local perceptions into a global perception [50]. This may explain why the autistic brain cannot properly recruit the frontal region for visual detection. However, in this kind of task that mainly requires processing of local aspect, this cognitive limitation may not have many adverse effects [19]. Rather, focusing on the global context can hinder finding a single detail.
In the aforementioned neuroimaging studies, TD individuals mainly activated the prefrontal region responsible for integration, but individuals with ASD did not. In contrast, the weak central coherence hypothesis, which considers visual signaling as being normal, cannot explain relative hyperactivation of the early visual cortices in ASD. Instead, according to the enhanced perceptual functioning hypothesis, individuals with ASD exhibit excessive low level perceptual processing, which is not regulated properly by top-down mechanisms and interferes with higher cognitive processes [20]. This is consistent with the fact that hyperactivation of the early visual cortex in ASD is observed not only in visuospatial tasks but also in social [6,51] or attention shifting tasks [52]. In addition, the autistic brain excessively activates the ventral occipitotemporal area in esthetic judgments that require subjective appreciation [21]. That is, in the autistic brain, low level visual processing occurs excessively even when it is unnecessary.

The cortical underconnectivity hypothesis argues that the cortical network between brain regions is inefficient, leading to impaired integration of information [53]. However, in terms of visual detection, the results from neuroimaging studies on connectivity are inconsistent. In one fMRI study using EFT, frontal-posterior underconnectivity was observed as expected [45]. In functional connectivity MRI (fcMRI) studies using BDT and visual search task, the inter-lobar connectivity of the ASD group was not different from that of the TD group [40], and the fronto-occipital connectivity was even increased in the ASD group [49]. Many task-based fcMRI studies have examined functional domains in which individuals with ASD show disabilities, but a visual detection task is exceptional in that individuals with ASD have comparative or superior performance. Task-related brain activity may have an additional effect on correlation between blood-oxygen-level-dependent (BOLD) signal fluctuations in certain brain regions, or functional connectivity [54]. Therefore, the impact of task selection should be considered when evaluating functional connectivity, and some underconnectivities reported in individuals with ASD may not reflect actual reduced integrity of neural networks [49].

\section{Do individuals with ASD exhibit extraordinary visual detection capability?}

As mentioned above, individuals with ASD showed atypical brain activation or connectivity while performing visual detection tasks. Is it, then, true that they have superior visual detection capability? A number of studies have not found differences in task performance between ASD and TD individuals. According to a meta-analysis by Samson et al. [55], only $31 \%$ of neuroimaging studies using visual stimuli showed differences in performance between groups. This may be because 
a visual detection task performed in a scanner is too easy to be able to reveal a performance difference. In a study that applied various tasks with a range of difficulties by changing set size and distractor composition, the ASD group, compared to the TD group, exhibited increased search efficiency [41]. Another possible explanation is that, because ASD symptomatology is heterogeneous, only some individuals with ASD may have superior visual detection capabilities. However, there is some evidence that it is not a matter of visual detection. The eye gaze pattern of individuals with ASD was analyzed while they were observing naturalistic scene images, and the results revealed that they focused better on local detail, such as contrast and color [56]. While performing BDT, they showed a different cognitive mechanism from that exhibited by TD individuals, regardless of their visuospatial ability [57]. Thus, it is reasonable to consider that individuals with ASD have a different visual processing style and capability of visual detection varies from normal to extremely high, such as in the Savant syndrome.

\section{Visual detection and social impairment}

Interestingly, there are studies that have reported the relationship between visual detection-related brain activity and social impairment in ASD. In an fMRI study conducted by Spencer et al. [48], the ASD group showed decreased activities in the posterior brain regions, including the left fusiform gyrus and left V3 (the associative visual cortex), in EFT versus control task condition as compared to the TD group. Brain activities in these regions showed negative correlation with impairments in reciprocal social interaction evaluated by Autism Diagnostic Observation Schedule-Generic (ADOS-G) [58]. This indicates that atypical visual processing may have a negative effect on eye gaze, joint attention, and facial expression, which are reciprocal social interactions dependent on visual information. However, hypoactivation of the posterior brain regions in ASD may be an unexpected result. Generally, in an fMRI study, task-related neural activity is derived by subtracting baseline BOLD signals from BOLD signals generated while performing the task. Researchers usually use BOLD signal generated while performing control tasks as the baseline; however, occasionally, resting state is also used as the baseline [59]. In the study conducted by Spencer et al. [48], a simple control task was used to determine whether a target shape is the same as a highlighted shape in a complex figure. If the ASD group overactivated the posterior brain regions even during a simple control task that does not require a visual search, comparison of EFT versus control task conditions of the two groups does not lead to the conclusion that the ASD group activated less the posterior brain regions. Instead, from this perspective, it is possible to conclude that excessive visual function is associated with more severe autistic symptoms. In a study conducted by Keehn et al. [49], no group difference in brain activity was observed during visual search, but neural activity of the right middle frontal gyrus was positively correlated with socio-communicative impairment and neural activity of the left intraparietal sulcus (IPS) was negatively correlated with socio-communicative impairment. These two regions are thought to be related to visual attention. Although there is still a lot to be learned, it is worth noting that the brain response to non-social visual stimuli may be related to social impairment in ASD.

In summary, individuals with ASD have shown superiority in the performance of visual detection tasks that require relatively little higher order visual processing. Although their task performance is not as good as expected, the findings from neuroimaging studies discussed above provide evidence that their visual perception is more dependent on early visual processing. If basic visual processing in ASD is atypical, it is plausible that it can affect processing of visually presented social stimuli, which requires integration and judgment of various information.

\section{MOTION PERCEPTION}

Humans live in a dynamic world, not in a still picture. Therefore, motion perception plays a fundamental role in perceiving their environment and determining their behavior. According to psychophysical studies, local motion perception of a single object in ASD seems to be unimpaired overall [60,61]. Some findings suggest that individuals with ASD exhibit normal first order motion perception, but impaired second order motion perception [60]. First order motion stimuli defined by luminance are easily identified from the background, but second order motion stimuli defined by features other than luminance (e.g., contrast, texture, etc.) cannot be identified from the background, unless they are moving, and this requires more complex motion processing in the brain [62]. Studies investigating perception of coherent motion, a type of global motion, have provided mixed results. In a coherent motion task usually represented in a random dot kinematogram (RDK), a certain percentage of dots move in the same direction while other dots move in random direction. An individual is required to identify the general direction of moving dots. For example, if 50\% of dots move in a random direction and the remaining 50\% of the dots move to the right, an individual can recognize that the general direction is right. Motion coherence threshold is measured by gradually increasing the coherence level (i.e., percentage of dots moving in the same direction among all dots). For example, an individual's motion coherence threshold is 
$30 \%$ if the individual first notices that the general direction is upward at a point where $30 \%$ of dots are moving upward and $70 \%$ of the dots are moving in random direction [63]. Multiple studies have reported coherent motion processing impairment in ASD [27,28], but there was no group difference between the ASD and TD groups after adjustment for intelligence quotient (IQ) [64]. In a recent behavioral study of coherent motion, performance of individuals with ASD was similar to or lower than that of TD individuals depending on viewing duration [65].

\section{Atypical global motion perception in ASD}

Visual information that reaches the primary visual cortex is divided into ventral stream and dorsal stream. The ventral stream receives input from the parvocellular pathway that processes high spatial frequency (HSF) and transmits it from V1, through V4, to the inferior temporal cortex, and is called "what" system as this stream processes visual form and color. The dorsal stream receives input from the magnocellular pathway that processes low spatial frequency (LSF) and transmits it from V1 and V2, through the middle temporal visual area (MT/V5), to the posterior parietal cortex, and is called "where" system as this stream processes location or movement of objects [66]. The dorsal stream is involved in global motion perception, and especially MT/V5 is considered a key region where local motion signals are integrated into a global representation [67]. Moreover, the neural response of MT/V5 appears to be enhanced as the coherence level increases [68].

The dorsal stream deficit hypothesis attempts to explain global motion perception impairment in ASD [28]. Children with dorsal stream dysfunction are unable to visually recognize multiple objects at the same time, so they cannot pick a toy they want from a toy box. They are unable to accurately recognize moving objects, making it difficult to count the number of fingers of a moving hand. They find it difficult to take the stairs without directly touching the next step, due to disrupted visual guidance of movement [69]. The fact that children with ASD can barely learn by simply watching without somatosensory feedback and show impaired imitative learning suggests the possibility of disrupted dorsal stream processing [70]. In addition, seeing a moving object through lateral glance is thought to be a compensatory behavior for children with ASD to easily perceive LSF information, which is processed inadequately due to dorsal stream deficit [71]. The weak central coherence hypothesis considers that global motion perception impairment observed in individuals with ASD is due to their general cognitive style that focuses on local detail, but not on global features [50]. Two prominent examples of global motion are coherent motion and biological motion, and these two types of motion have been used to evaluate global motion perception.

\section{Coherent motion perception}

Some fMRI studies have investigated whether the dorsal stream deficit hypothesis is applicable to coherent motion perception in individuals with ASD. In an fMRI study by Brieber et al. [72], participants performed coherent motion tasks represented in RDK while they were scanned. Activity of V5 in the TD group was greater while they were viewing coherent motion compared to random motion, whereas this difference was not found in the ASD group; that is, activity of V5, which is a key region of the dorsal stream, was not modulated by motion coherence. The ASD group showed hypoactivation of the superior parietal cortex in coherent motion versus random motion contrast. The authors argued that unmodulated activation in lower dorsal stream results in lack of motion coherence-specific activation, and hypoactivation of the parietal cortex prevents motion signals from being integrated into global perception. In addition, the ASD group showed hyperactivation of V1. In contrast, another study that applied a similar design showed that both the ASD and TD groups recruited MT/V5 and no differential neural activity was found between the two groups, although the ASD group exhibited a non-significant trend toward lower performance in the coherent motion task [73].

Multiple studies have investigated the coherent motion perception in individuals with ASD, but their task performances have shown mixed results. In this regard, the following study is notable. Robertson et al. [74] instructed individuals to choose a global direction, showing RDKs with varying coherence levels and viewing durations while scanned. As both variables decreased, the performance level declined more sharply in ASD than in TD individuals. Similarly, ASD individuals showed greater reduction in V1 and MT/V5 activity compared to TD individuals as viewing duration decreased. Moreover, taskrelated activities in these early visual areas showed strong positive correlation with autistic symptomatology. A fast-paced real world where instantaneous perception and judgment is necessary may be a challenging environment for individuals with ASD. There was no group difference in IPS activity. The results of this study indicated that coherent motion perception per se is not impaired, but is heavily impacted by motion signal strength, and this impact occurs at the early visual cortex level. The authors argued that this exaggerated impact may arise from failure of local opponent inhibition, which is related to imbalance of excitatory/inhibitory neural transmission. In another study, individuals with ASD showed greater activation and faster hemodynamic decay in V5 while passively viewing outward moving rings. This finding also suggested an atypical excitation/inhibition pattern in ASD [75]. 
A magnetoencephalography (MEG) study [76] using RDK provided more direct evidence for the imbalance of excitatory/inhibitory neural transmission in ASD. This study investigated gamma-band activity of visual areas for various motion coherences. Gamma-band is generated by a local loop in which gamma aminobutyric acid (GABA)-A receptor and excitatory receptor are alternately activated [77], and is related to local excitatory-inhibitory interaction in the cortex [78]. Activity of gamma-band is thought to increase as visual motion strength increases [79]. Gamma-band activity of motionresponsive areas V3, MT/V5, and V6 increased as motion coherence increased in both the ASD and TD groups. In other words, motion coherence caused positive linear modulation of gamma-band activity. However, this positive modulation was stronger in the ASD group (Fig. 1), showing that local imbalance of excitation/inhibition may be associated with impaired coherent motion perception in ASD. One magnetic resonance spectroscopy study showed that binocular rivalry, which relies on visual inhibitory signals, was reduced in ASD, and the reduction was associated with a decrease in GABA concentration in the early visual cortex [80]. The imbalance of excitation/inhibition in the autistic brain may account for dysfunction of lateral inhibition [81] and increased internal noise [82] in early visual processing in ASD and is consistent with observations that individuals with ASD often show inter-trial variability of neural responses [83].

\section{Biological motion perception}

Biological motion, a type of global motion, refers to movement of a person or an animal and exhibits a complex temporal and spatial pattern. Nevertheless, two-day-old infants show preferential looking at biological motion [84] and three-month old infants distinguish between biological and non-biological motion expressed by a point-light display (PLD) [85]. Preferential attention to biological motion plays an important role in social development [86], but unlike TD toddlers, autistic toddlers focus on non-social motion rather than biological motion [87]. PLD is most frequently used for biological motion research. It displays movement of human major joints as dots and is free from confound of form since it has no shape [88]. Scrambled motion is often used as a control stimulus. It is composed by changing the position of each dot while local movements remain the same as the biological motion. Although individuals with ASD show relatively preserved ability of basic information extraction such as simple identification from biological motion, they have difficulties in obtaining complex and ambiguous information such as emotional contents [89] or distinguishing biological motion from scrambled motion [90].

The superior temporal sulcus (STS), a brain structure highly connected to social brain network [91], has shown altered ac-

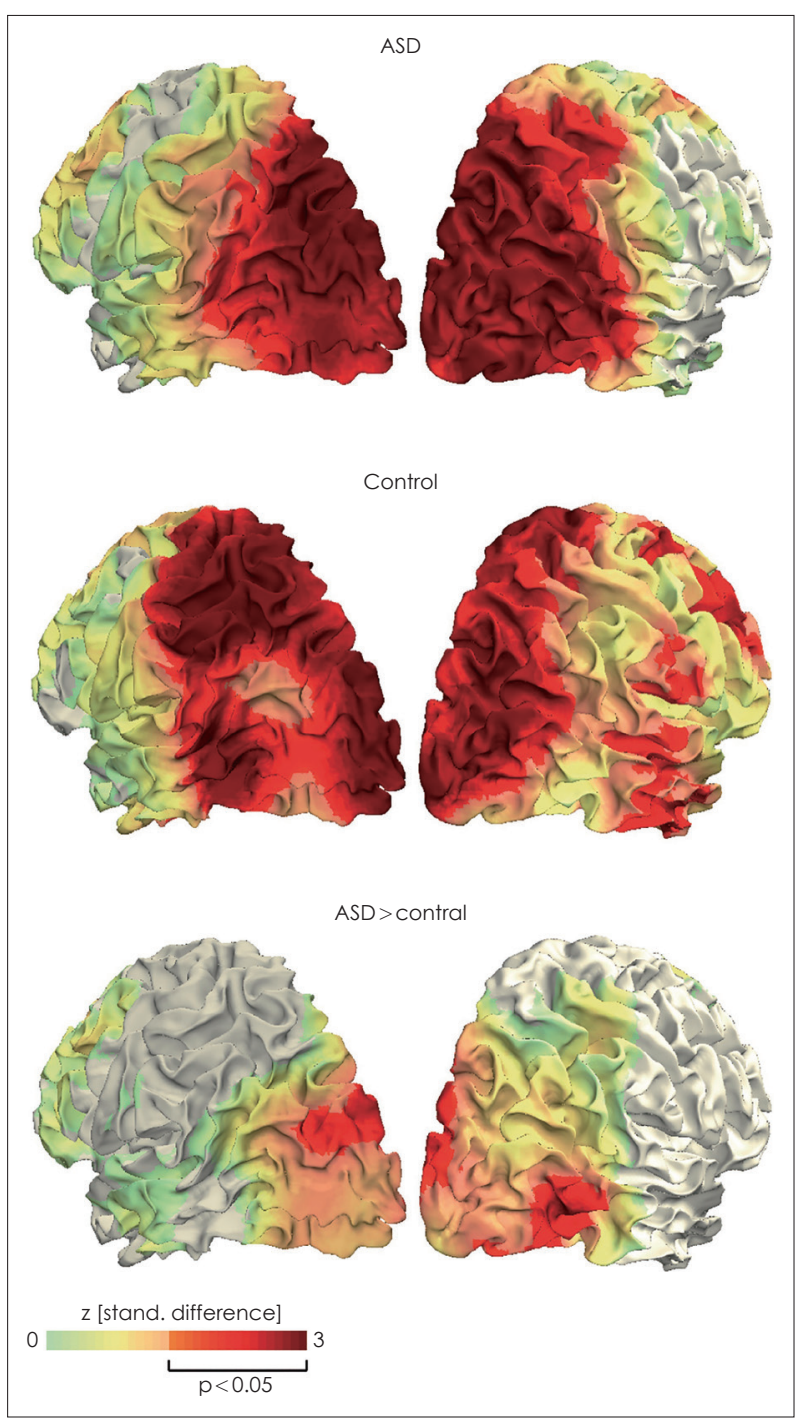

Fig. 1. Cortical distribution of the positive linear modulation of gamma-band activity by motion coherence. Two upper figures show that both group exhibited this modulation mainly in visual areas. Lower figure shows that the stronger stimulus-related gamma-band modulation in ASD occurred from extrastriate areas including V3, MT/V5 and V6. ASD: autism spectrum disorder. Adapted from Peiker et al. PLoS One 2015;10:e0132531 [76].

tivities or connectivities during socio-emotional perception in ASD [92,93]. Moreover, it has been speculated that the STS is connected to multiple primary sensory cortices and receives sensory information including non-social information [94]. STS seems to be involved in biological motion processing, given that social meaning must be provided to visual information in order to interpret biological motion. In fact, several neuroimaging studies investigating neural response to biological motion expressed in PLD have reported abnormalities of STS, particularly posterior STS (pSTS) in ASD.

In a study by Herrington et al. [95], participants were required to choose walking direction of point-light walker dur- 
ing fMRI scanning, and no behavioral difference was observed between the Asperger syndrome group and the TD group. However, while individuals with Asperger syndrome showed less activity only in the adjacent region of the inferior parietal lobe while viewing scrambled walkers compared to the TD group, they showed extensive hypoactivation in the right middle and inferior temporal regions and the superior temporal gyrus including STS while viewing intact walkers. Similarly, in another fMRI study, individuals with ASD hypo-activated the middle temporal cortex close to the STS while they were choosing walking directions of point-light walkers [96]. In a study examining the neural activity of the ASD group, compared to the unaffected sibling (US) group, and TD group [97], individuals were asked to simply watch motion clips during scanning. The ASD group showed decreased activity in the prefrontal and temporal cortices including the right PSTS in biological motion versus scrambled motion contrast, compared to the US and TD groups. Furthermore, the activity of the right pSTS was negatively correlated to severity of social deficit. The authors argued that the right pSTS shows "state activity" as a biological marker indicating the severity of autistic conditions. It is noteworthy that even in the process of extracting or simply observing basic information of neutral PLD stimuli that do not reveal emotion or intention, the autistic brain showed atypical neural activities. This suggests that the characteristics of biological motion perception in ASD cannot be explained only from the perspective of social impairment.

In one fMRI study [73], ASD individuals showed lower task performances and hypoactivation of pSTS, DLPFC, and IPS. The authors noted the possibility that PLD stimuli with noise caused difficulties in dynamic attention, although they did not investigate this hypothesis directly. Indeed, there have been reports that individuals with ASD performed better than TD individuals in noiseless motion paradigm [98]. Therefore, individuals with ASD may not have difficulties in integrating information, but only in combination with signal and noise. In another fMRI study [99], participants were instructed to look at biological motion or scrambled motion and choose whether the presented PLD was a person or not (explicit task), or to indicate color change of moving dots (implicit task). In implicit task, both the ASD group and the TD group equally recruited the right STS. However, in explicit versus implicit contrast, ASD participants, unlike TD participants, did not show augmentation of right STS activity and the degree of augmentation was correlated with explicit task performance. This result showed that individuals with ASD may lack the ability to attend to social stimuli despite their intact basic biological motion processing, which is consistent with the social motivation theory of ASD.

Lastly, the cerebellum is functionally connected to many ce- rebral cortices responsible for social and sensory functions, and abnormal functions of the cerebellum have also been reported in ASD [18]. The cerebellum generates a prediction model based on experience of cognitive results of cerebral activities, so that we can perform complex skills easily and immediately away from slow and hard top-down processes $[100,101]$. In particular, we instinctively recognize biological motion faster than any other visual information [95]. One recent fMRI study [100] investigated how the cerebellum contributes to biological motion perception in autism and typical development. While viewing point-light biological motion, the cerebellar posterior lobe was activated in both groups, and no significant group difference in activity was found. However, effective connectivity between cerebellar posterior lobe regions and the right pSTS, a key region of biological motion perception and cerebellar posterior lobe regions, was correlated with greater autistic symptomaticity. This indicates that the role of the cerebellum in biological motion perception in ASD should be investigated in future studies.

Individuals with ASD have shown altered brain response in perceiving coherent motion and biological motion, which are representative global motions. While perceiving coherent motion, these individuals show atypical activation of the primary visual cortex and extrastriate cortices, including the MT/V5 region, which is thought to be a key region for global motion processing. These atypical neural responses may be attributable to the imbalance of excitation/inhibition. The results of these studies suggest abnormal early visual processing in ASD, and cannot explain whether atypical motion perception is due to impaired general cognitive function. Regarding the biological motion perception, individuals with ASD have shown altered activation of STS. This may be interpreted as reflecting social impairment such as lack of social motivation but may also be regarded as having difficulty of external noise filtering [102]. Future research should investigate how the autistic brain responds to biological motion surrounded by noise, which better reflects real life. If an atypical response is found, it will be necessary to explore whether it is a matter of visual processing or of a more general domain.

\section{FACE PROCESSING}

Human face is the most commonly encountered social stimulus in real world. From faces, we obtain various social information in an immediate and instinctive manner [103]. However, face processing in the brain is complicated. First, individual local features such as eye, nose, and mouth are identified, and they are holistically perceived through configural processing. Then, the local and global motions of tem- 
porally and spatially dynamic facial structures are analyzed. Lastly, all this information is synthesized to derive a social meaning. Maturation of face processing, which requires a high degree of sophistication, is completed in late development [103]. For example, major brain regions involved in face processing, inferior occipital gyrus (IOG) and fusiform gyrus (FG), begin to show stronger activation on faces than objects only at ages 11-14 [104].

Behaviorally, individuals with ASD have shown difficulties in face recognition and discrimination [31], face memory [105], gender discrimination [32], emotional expression perception [106], and diminished attention to eyes, which is the most informative feature [107]. It is not surprising that numerous studies have investigated neural correlates of face processing in autistic individuals, since social impairment is the main problem in ASD. Most studies have examined the response of cerebral cortex to static face stimuli and have reported atypical responses of the brain. However, because many studies have been conducted, it is also true that they have produced mixed results. The fact that development of face processing is completed at a relatively late age and that individuals with ASD show a heterogeneous spectrum could be one reason of inconsistency. In addition, gaze direction, emotional expression and familiarity of faces affect neural activity [35]. Herein, we summarize the main findings of studies that have investigated face processing in ASD for the past 20 years. These studies have mainly focused on cortical responses to static face stimuli. Next, we report findings from neuroimaging studies that have examined dynamic face processing and subcortical face processing, both of which have been investigated recently.

\section{Static face processing}

According to the human neural network for face processing, proposed by Haxby et al. [108], IOG and FG mediate visual analysis of invariant or unchangeable features (e.g., gender, identity), and STS and amygdala mediate visual analysis of variant or changeable features (e.g., emotional expression, eye gaze direction). Specifically, it seems that IOG mainly analyzes individual facial features such as eyes or noses [109], and FG is involved in more complex processing such as integrating individual features [110]. STS is thought to analyze dynamic aspects of faces [111], and the amygdala is a key region of emotional expression perception [112].

In general, individuals with ASD, compared to TD individuals, showed hypoactivation in IOG, FG, STS and amygdala [113-116]. The most consistent finding is hypoactivation of FG, which is supposed to be a general deficit of face processing in ASD [103]. In Pierce and Redcay's fMRI study [117], children with ASD showed decreased activity in the FG while looking at pictures of strangers, but the decreased activity was normalized when looking at pictures of familiar people. Familiarity can influence engagement of FG, and decreased activity of FG in ASD may reflect reduced attention to strangers. The amygdala exhibited lower activity in response to neutral faces in individuals with ASD compared to TD individuals $[114,115]$. Interestingly, activity of this region seemed to depend on where eye gaze was being fixed. In other words, it was found that activity of the amygdala had a positive correlation with time spent in eye-region fixation [34]. While natural viewing, individuals with ASD showed less gaze toward eye-region, and this behavioral feature was associated with a threat of neutral faces. Moreover, forcing eye-region fixation enhanced the activity of amygdala in individuals with ASD [118]. In an fMRI study that participants were watching facial expressions while scanned, a gender discrimination task was performed to maintain concentration. As a result, the ASD group showed increased activity in the amygdala compared to the TD group [119]. These findings indicate that individuals with ASD are likely to avoid eye contact because it could increase their arousal [120]. Activities of occipital gyrus or STS during face processing in ASD are inconsistent. In particular, studies using faces with emotional expressions showed more mixed results. This may be because each facial emotion (e.g., anger, disgust, fear, happiness, sadness, etc.) affected neural activities in different ways. The autistic brain, compared to the TD brain, showed either higher [121], or lower [122] activation of STS, in response to faces with emotional expression, and activity in the occipital cortex showed similar results $[122,123]$.

\section{Dynamic face processing}

In dynamic real world, we do not interact with pictures. A number of neuroimaging studies have investigated face processing in ASD, but one of the biggest limitations is the use of static pictures. Using static stimuli, the effects of motion perception, in which individuals with ASD are thought to show impairment, cannot be examined. It was recently discovered that TD individuals exhibit higher activity in several cortical regions, including pSTS, when looking at dynamic faces compared to static faces [124]. Considering movements of faces, Bernstein and Yovel [125] proposed an integrative neural model for dynamic and static face processing (Fig. 2). According to their model, the dorsal face areas including the pSTS and inferior frontal gyrus (IFG) are responsible for processing facial motion or changeable aspects of faces. The ventral face areas including occipital face area (OFA) and fusiform face area (FFA) are involved in facial form processing, which suggests that these areas process variant and invariant facial features, regardless of whether the face is moving. The motion- 


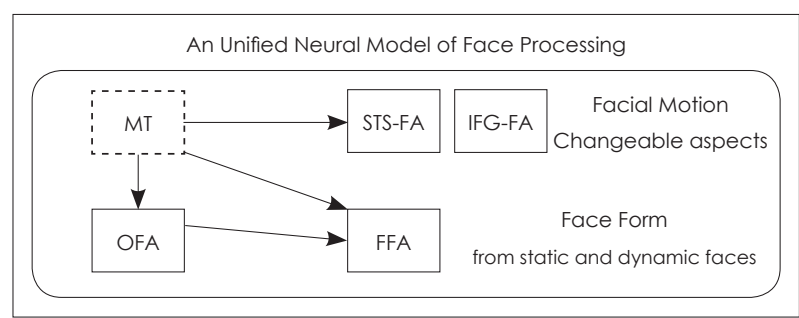

Fig. 2. The unified neural model of face processing. MT sends input to the dorsal face areas (i.e., STS-FA and IFG-FA) for facial motion processing, and to the ventral face areas (i.e., OFA and FFA) for structure-from-motion analysis. The dorsal face areas are responsible for facial motion and changeable facial aspects, while the ventral face areas are responsible for facial form processing of static and dynamic faces. MT: middle temporal visual area, STS-FA: superior temporal sulcus face area, IFG-FA: inferior frontal gyrus face area, OFA: occipital face area, FFA: fusiform face area. Reproduced from Bernstein et al. Sci Rep 2018;8:7036 [126].

selective region MT/V5 is connected to both the dorsal and ventral face areas. This region sends motion information to pSTS and structural information to OFA and FFA in motionless form [126].

Pelphrey et al. [127] conducted an fMRI study using moving dynamic face stimuli for the first time in ASD research. Participants watched static and dynamic facial stimuli with or without emotional expressions while they underwent scanning. In both the ASD group and TD group, the amygdala, FG, and STS were more activated in response to dynamic emotional expressions than in response to static emotional expressions. However, these three regions in the ASD group showed reduced positive modulation of activities by dynamic faces. Neural response of MT/V5 to dynamic faces was not different between the two groups. Thus, the authors concluded that individuals with ASD have impairment in dynamic face processing and abnormalities in social brain regions such as the amygdala and STS may have negative effects on dynamic face processing in ASD. On the other hand, they argued that the early visual area such as MT/V5 in ASD is intact. An fMRI study by Sato et al. [111] showed similar results (Fig. 3). Additionally, dynamic causal modeling analyses revealed that bidirectional effective connectivity was found in V1-middle temporal gyrus (MTG) circuit and MTG-IFG circuit in both the ASD group and control (CON) group, and all of these effective connectivities increased in response to dynamic faces compared to static faces. However, the enhancements of effective connectivities were significantly weaker in the ASD group than in the CON group (Fig. 4). These results suggested that dynamic face processing in ASD is disrupted from early visual processing (i.e., V1-MTG). One recent study [128] provided evidence that individuals with ASD may have difficulties in face-to-face communication. Participants performed visual speech recognition tasks by matching an articulated syllable with a target syllable while watching a silent video of speakers articulating a syllable. Control tasks were face identification. While both the ASD group and TD group showed functional connectivities between dorsal movement regions and ventral form regions in visual speech recognition versus face identification contrast, the ASD group showed reduced connectivity in right MT/V5-right OFA circuit and the left temporal visual speech area (i.e., the portion of pSTS which is specifically sensitive to visual speech)-left FFA circuit. This result indicated that individuals with ASD may suffer from interpersonal communication difficulties due to atypical dynamic face processing. In contrast, there was no difference in cortical activity between individuals with ASD and TD individuals while viewing dynamic facial expression in one study [129].

\section{Subcortical face processing}

Based on a number of electrophysiological, neuropsychological and neuroimaging studies, Johnson [130] concluded that humans are characterized by a subcortical face detection pathway. This pathway runs from the superior colliculus to the amygdala via the pulvinar of the thalamus, which transmits visual information to the amygdala faster than through the visual cortex. Fig. 5 shows the cortical and subcortical pathways schematically. Newborn babies are not fully equipped with a cortical visual system that delivers HSF information containing the fine details of objects. Inevitably, they rely much more on the subcortical visual system that delivers LSF information containing coarse aspects of objects. Even under this condition, babies are able to preferentially look at face-like patterns. This is consistent with the fact that a fearful face with open eyes and a mouth filtered by LSF can even activate a subcortical face processing system including the amygdala [131]. Importantly, it is thought that preference for face-like patterns at early development activates the lateral occipital, fusiform and orbitofrontal cortex to help mature cortical face processing [130]. In short, a subcortical face processing system plays an important role in enabling very basic face perception through the rapid delivery of crude but essential information and developing face processing. One-year-old infants with ASD do not look at faces as much as TD infants, and this could be an early sign of abnormal face processing [132]. In addition, dysfunction of the amygdala, an important region of the subcortical face processing system, can reduce social motivation and prevent proper attendance to socially relevant stimuli, resulting in face processing abnormality [133]. Taken together, it is necessary to investigate characteristics of subcortical face processing in the autistic brain to reveal neurobiological underpinnings of ASD.

Faces filtered by HSF and LSF have often been used to evaluate subcortical face processing, and this type of stimuli was 

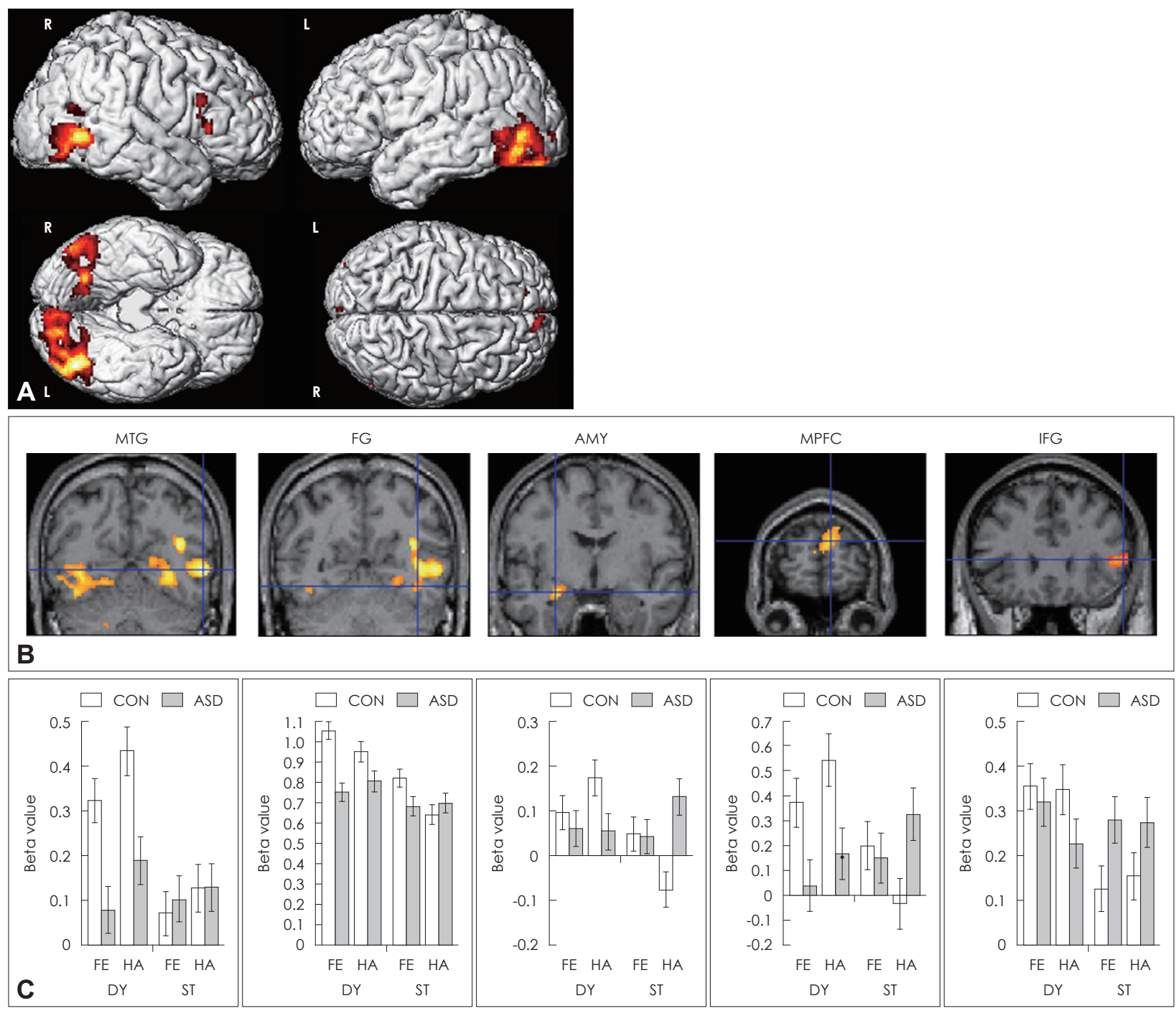

Fig. 3. Brain regions in which ASD group showed reduced activity compared to CON group under dynamic versus static conditions. (A) Statistical parametric maps rendered on spatially normalized brains. (B) Statistical parametric maps of representative brain regions overlaid on the normalized anatomical MRI of one of the participants. (C) Mean parameter estimates ( \pm SE) of brain regions corresponding to the above overlaid MRIs. MTG: middle temporal gyrus, FG: fusiform gyrus, AMY: amygdala, MPFC: medial prefrontal cortex, IFG: inferior frontal gyrus, FE: fear, HA: happiness, DY: dynamic expressions, ST: static expressions, CON: control, ASD: autism spectrum disorder. Adapted from Sato et al. BMC Neurosci 2012;13:99 [111].

also used in studies on disorders other than ASD (Fig. 6) [134]. Vlamings et al. [135], in their visual evoked potential study, showed LSF and HSF images of fearful and neutral faces to 3 to 4-year-old children with or without ASD. In TD children, P1 activity in the visual area was observed to be modulated by fearful faces only when they were presented in LSF, but not in HSF. In contrast, in children with ASD, this effect occurred only when the faces were presented in HSF, but not in LSF. Based on these results, the authors proposed that the subcortical face processing route that is responsible for perception of emotional expression in children with ASD is impaired, leading to excessive dependence on HSF information from an early age. This study provided evidence that atypical face processing in adults with ASD may originate from disrupted subcortical processing in early age. In another fMRI study [136] conducted in adult participants, similar face stimuli were used. In the TD group, fusiform activity increased by emotional expression regardless of spatial frequency, but the ASD group showed an increase in fusiform activity only by LSF faces. The amygdala in the ASD group was more sensitive to LSF stimuli than HSF as opposed to the TD group. The authors concluded that individuals with ASD exhibited preserved subcortical LSF face processing, but cortical analysis of HSF face was abnormal. Although the results of the two studies seem to contradict each other, from a neurodevelopmental perspective, impaired subcortical face processing in infants 3-4 years old 
may lead to abnormal cortical face processing in adulthood. A limitation is that the two studies did not directly examined subcortical activity. To draw more consistent conclusions, it is necessary to directly examine structural or functional characteristics of the subcortical face processing system.

Several studies have used subliminal face stimuli to investigate subcortical face processing in ASD. Subliminal face stimuli are presented for a very short time (approximately 20-50 ms) [137], and usually followed immediately by backward masking. The former activates subcortical regions, especially amygdala, but is not consciously perceived by the cerebral cortex [138]. In an fMRI study by Kleinhans et al. [139], direct group comparison revealed that no brain region showed higher activation the ASD group than in the TD group. The TD group showed higher activation than the ASD group in the left amygdala, bilateral FG, right pulvinar, and bilateral superior colliculi. This result suggested impaired subcortical face processing in ASD. In contrast, in another study, there was no group difference in time-course or intensity of amygdala activation between the ASD and the TD group when subliminal fearful faces were presented [140]. Altered subcortical face processing could be implicated in gaze avoidance observed

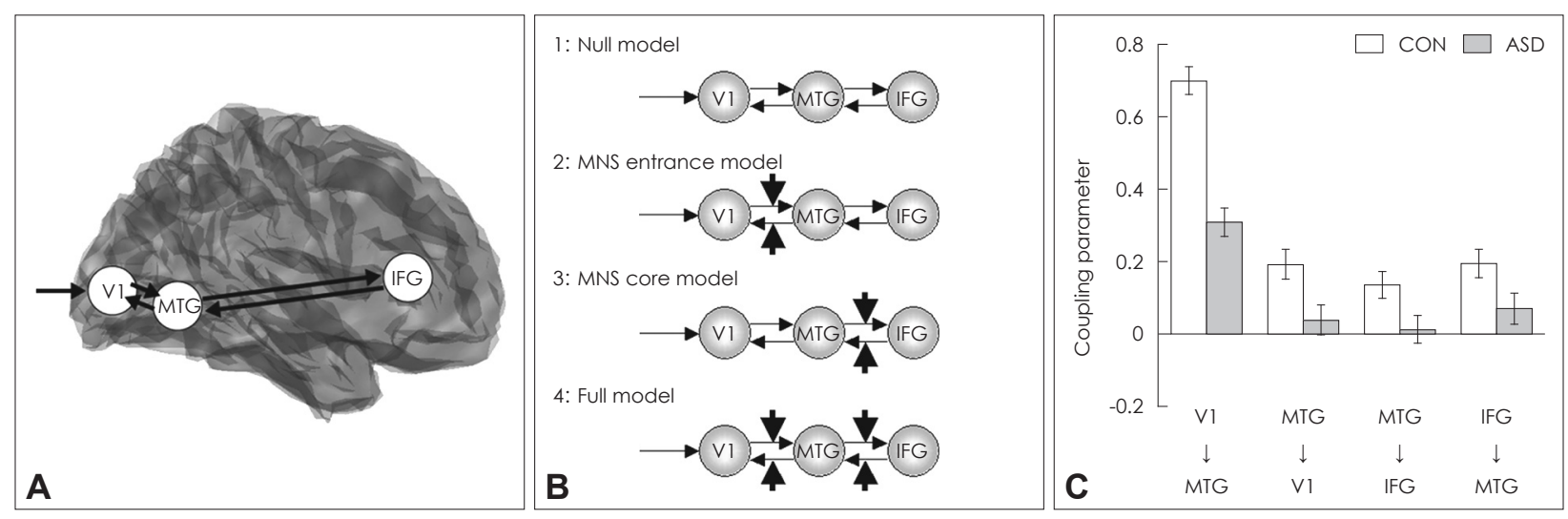

Fig. 4. Models and results of dynamic causal modeling analyses. (A) Bidirectional intrinsic connections constructed between the $\mathrm{V} 1$ and MTG and between the MTG and IFG. (B) Models based on the modulatory effect (bold arrows) of dynamic presentation. The full model was the best fitted for both groups. (C) Mean coupling parameters ( \pm SE) for CON and ASD group. V1: primary visual cortex, MTG: middle temporal gyrus, IFG: inferior frontal gyrus, MNS: mirror neuron system (i.e., MTG-IFG), CON: control, ASD: autism spectrum disorder. Adapted from Sato et al. BMC Neurosci 2012;13:99 [111].

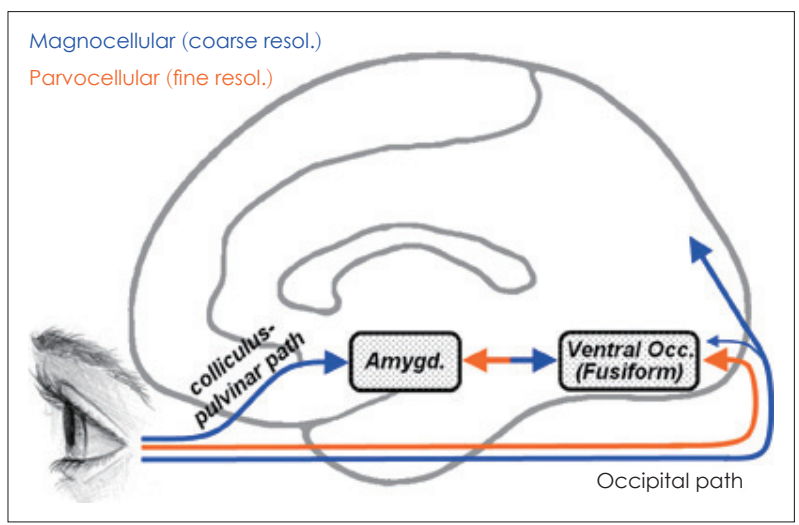

Fig. 5. Cortical and subcortical pathways for visual inputs. Orange arrows describe parvocellular pathways conveying fine (high spatial frequency) visual information, whereas blue arrows describe magnocellular pathways conveying coarse (low spatial frequency) information. Parvocellular inputs reach the striate cortex and project to ventral occipital regions, including the fusiform gyrus. Magnocellular inputs project more dorsally toward the parietal cortex and, to a lesser extent, also toward the ventral occipital cortex. Another magnocellular pathway reaches the amygdala via a subcortical colliculus-pulvinar projection. Fusiform and amygdala are reciprocally connected. resol.: resolution, Amygd.: amygdala, Occ.: occipital cortex. Reproduced from Corradi-Dell'acqua et al. Front Hum Neurosci 2014;8:189 [136].

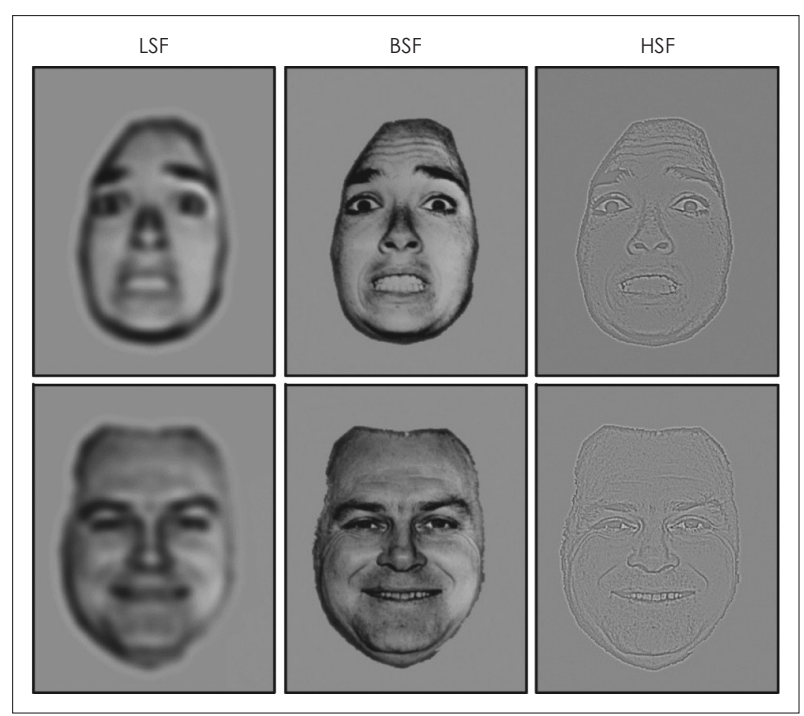

Fig. 6. Examples of face stimuli sorted by spatial frequency. Faces with normal presentation contains broad spatial frequency. These faces are spatially filtered to form LSF and HSF face stimuli. BSF: broad spatial frequency, LSF: low spatial frequency, HSF: high spatial frequency. Adapted from Celeghin et al. Biomed Res Int 2019;2019:9562935 [134]. 
in ASD. In one study [141], forced gaze fixation to eye-region on dynamic emotional face caused hyperactivation of the pulvinar and amygdala to individuals with ASD. Especially, activity in the amygdala was not affected by emotional valence of faces. These results indicated that individuals with ASD may avoid looking in the eyes with emotional expression to prevent hyperarousal.

The results of research on face processing in ASD can be summarized as follows. Since faces are social stimuli, abnormalities of face processing in ASD are well explained on the basis of impaired social brain networks. In fact, face perception in individuals with ASD is accompanied by atypical responses in social brain regions. However, atypical brain responses to dynamic faces appear in the early visual cortex, including MT/V5. This indicates the possibility that failure to perceive complex movements of faces is implicated in atypical face processing in ASD. ASD is usually diagnosed after two years of age [142], but high-risk infants already do not show a preference for faces when they are one year old [132]. Considering that this preference in infants is related to subcortical face processing, neurobiology of face processing in ASD may be underpinned by altered subcortical face processing to some extent. There are not many neuroimaging studies that have investigated dynamic face processing or subcortical face processing in ASD, and the results are inconsistent.

\section{CONCLUSION}

Findings from neuroimaging studies in ASD provide some evidence for altered or disrupted visual perception in ASD. Multiple pieces of evidence indicate that individuals with ASD show abnormalities in early visual processing before higher order cognitive or perceptual processing. Specifically, the autistic brain shows atypical activity or connectivity in the primary visual cortex and the extrastriate cortex including MT/ V5 in response to various visual stimuli. If early visual processing in ASD is atypical per se, perception of human faces showing complex changes in time and space can also be affected regardless of social impairment, a key symptom of ASD. Individuals with ASD exhibit atypical neural activity in the early visual cortex while viewing dynamic faces and may show abnormalities in subcortical face processing. Importantly, atypical visual perception can be closely related to social impairment in ASD. Altered visual perception precedes the diagnosis leading to the hypothesis that atypical visual processing may give rise to or aggravate social impairment. Moreover, several neuroimaging studies on ASD showed that atypical neural activity in ASD for various visual stimuli was associated with social impairment. Presumably, individuals with ASD may fail to process visual information properly, including social information, and isolate themselves from others as they feel confused about the visually presented social cues. In contrast, some individuals with ASD excel in artistic fields such as drawing. Visual perception in ASD may be a strength or weakness depending on whether its characteristics are accurately identified and utilized or not. In conclusion, to improve rehabilitation and quality of life of individuals with ASD, future studies are required to reveal characteristics and neurobiology of visual perception in ASD.

\section{Acknowledgments}

None.

\section{Conflicts of Interest}

The authors have no potential conflicts of interest to disclose.

\section{Author Contributions}

Conceptualization: Seungwon Chung, Jung-Woo Son. Data curation: Seungwon Chung, Jung-Woo Son. Investigation: Seungwon Chung. Project administration: Jung-Woo Son. Supervision: Jung-Woo Son. Validation: Jung-Woo Son. Visualization: Seungwon Chung. Writing_original draft: Seungwon Chung. Writing_review \& editing: Seungwon Chung, Jung-Woo Son.

\section{ORCID iDs}

Seungwon Chung https://orcid.org/0000-0002-3009-2722

Jung-Woo Son https://orcid.org/0000-0003-4972-3923

\section{REFERENCES}

1) American Psychiatric Association. Diagnostic and statistical manual of mental disorders (DSM-5). 5th ed. Arlington, VA: American Psychaitric Association;2013.

2) Brothers L. The neural basis of primate social communication. Motiv Emot 1990;14:81-91.

3) Sato W, Uono S. The atypical social brain network in autism: advances in structural and functional MRI studies. Curr Opin Neurol 2019;32:617-621.

4) Son JW, Ghim HR. Broken mirror or unbroken mirror?; an investigation for mirror neuron dysfunction of the autism spectrum disorder. J Korean Acad Child Adolesc Psychiatry 2013;24:109-123.

5) Kim SY, Choi US, Park SY, Oh SH, Yoon HW, Koh YJ, et al. Abnormal activation of the social brain network in children with autism spectrum disorder: an fMRI study. Psychiatry Investig 2015; 12:37-45.

6) Chung S, Son JW, Lee S, Ghim HR, Lee SI, Shin CJ, et al. Neural correlates of cognitive and emotional empathy in patients with autism spectrum disorder. J Korean Acad Child Adolesc Psychiatry 2016;27:196-206.

7) Tomchek SD, Dunn W. Sensory processing in children with and without autism: a comparative study using the short sensory profile. Am J Occup Ther 2007;61:190-200.

8) Estes A, Zwaigenbaum L, Gu H, St John T, Paterson S, Elison JT, et al. Behavioral, cognitive, and adaptive development in infants with autism spectrum disorder in the first 2 years of life. J Neurodev Disord 2015;7:24.

9) Baranek GT, Watson LR, Boyd BA, Poe MD, David FJ, McGuire L. Hyporesponsiveness to social and nonsocial sensory stimuli in children with autism, children with developmental delays, and typically developing children. Dev Psychopathol 2013;25:307-320.

10) Gliga T, Bedford R, Charman T, Johnson MH; BASIS Team. En- 
hanced visual search in infancy predicts emerging autism symptoms. Curr Biol 2015;25:1727-1730.

11) Robertson CE, Baron-Cohen S. Sensory perception in autism. Nat Rev Neurosci 2017;18:671-684.

12) Dellapiazza F, Michelon C, Oreve M, Robel L, Schoenberger M, Chatel C, et al. The impact of atypical sensory processing on adaptive functioning and maladaptive behaviors in autism spectrum disorder during childhood: results from the ELENA cohort. J Autism Dev Disord 2020;50:2142-2152.

13) Haupt $C$, Huber AB. How axons see their way--axonal guidance in the visual system. Front Biosci 2008;13:3136-3149.

14) Leekam S, Baron-Cohen S, Perrett D, Milders M, Brown S. Eyedirection detection: a dissociation between geometric and joint attention skills in autism. Br J Dev Psychol 1997;15:77-95.

15) Iacoboni M. Imitation, empathy, and mirror neurons. Annu Rev Psychol 2009;60:653-670.

16) Ozonoff S, Iosif AM, Baguio F, Cook IC, Hill MM, Hutman T, et al. A prospective study of the emergence of early behavioral signs of autism. J Am Acad Child Adolesc Psychiatry 2010;49:256-266.

17) Ozonoff S, Macari S, Young GS, Goldring S, Thompson M, Rogers SJ. Atypical object exploration at 12 months of age is associated with autism in a prospective sample. Autism 2008;12:457-472.

18) Thye MD, Bednarz HM, Herringshaw AJ, Sartin EB, Kana RK. The impact of atypical sensory processing on social impairments in autism spectrum disorder. Dev Cogn Neurosci 2018;29:151-167.

19) Shah A, Frith U. An islet of ability in autistic children: a research note. J Child Psychol Psychiatry 1983;24:613-620.

20) Mottron L, Dawson M, Soulières I, Hubert B, Burack J. Enhanced perceptual functioning in autism: an update, and eight principles of autistic perception. J Autism Dev Disord 2006;36:27-43.

21) Park SK, Son JW, Chung S, Lee S, Ghim HR, Lee SI, et al. Autism and beauty: neural correlates of aesthetic experiences in autism spectrum disorder. J Korean Acad Child Adolesc Psychiatry 2018; 29:101-113.

22) Son JW, Lee S, Jung WH, Jee SH, Jung SH. What is neuroaesthetics?: a new paradigm in psychiatry. J Korean Neuropsychiatr Assoc 2013;52:3-16.

23) Kaland N, Mortensen EL, Smith L. Disembedding performance in children and adolescents with Asperger syndrome or high-functioning autism. Autism 2007;11:81-92.

24) Bertone A, Mottron L, Jelenic P, Faubert J. Enhanced and diminished visuo-spatial information processing in autism depends on stimulus complexity. Brain 2005;128:2430-2441.

25) Kéita L, Guy J, Berthiaume C, Mottron L, Bertone A. An early origin for detailed perception in autism spectrum disorder: biased sensitivity for high-spatial frequency information. Sci Rep 2014; 4:5475.

26) Johansson G, von Hofsten C, Jansson G. Event perception. Annu Rev Psychol 1980;31:27-63.

27) Milne E, Swettenham J, Hansen P, Campbell R, Jeffries H, Plaisted K. High motion coherence thresholds in children with autism. J Child Psychol Psychiatry 2002;43:255-263.

28) Spencer J, O’Brien J, Riggs K, Braddick O, Atkinson J, WattamBell J. Motion processing in autism: evidence for a dorsal stream deficiency. Neuroreport 2000;11:2765-2767.

29) Vandenbroucke MW, Scholte HS, van Engeland H, Lamme VA, Kemner C. Coherent versus component motion perception in autism spectrum disorder. J Autism Dev Disord 2008;38:941-949.

30) Atkinson AP. Impaired recognition of emotions from body movements is associated with elevated motion coherence thresholds in autism spectrum disorders. Neuropsychologia 2009;47:3023-3029.

31) Dawson G, Carver L, Meltzoff AN, Panagiotides H, McPartland J, Webb SJ. Neural correlates of face and object recognition in young children with autism spectrum disorder, developmental delay, and typical development. Child Dev 2002;73:700-717.
32) Njiokiktjien C, Verschoor A, de Sonneville L, Huyser C, Op het Veld V, Toorenaar N. Disordered recognition of facial identity and emotions in three Asperger type autists. Eur Child Adolesc Psychiatry 2001;10:79-90.

33) Teunisse JP, de Gelder B. Impaired categorical perception of facial expressions in high-functioning adolescents with autism. Child Neuropsychol 2001;7:1-14.

34) Dalton KM, Nacewicz BM, Johnstone T, Schaefer HS, Gernsbacher MA, Goldsmith HH, et al. Gaze fixation and the neural circuitry of face processing in autism. Nat Neurosci 2005;8:519-526.

35) Klin A. Three things to remember if you are a functional magnetic resonance imaging researcher of face processing in autism spectrum disorders. Biol Psychiatry 2008;64:549-551.

36) Bölte S, Hubl D, Dierks T, Holtmann M, Poustka F. An fMRIstudy of locally oriented perception in autism: altered early visual processing of the block design test. J Neural Transm (Vienna) 2008;115:545-552.

37) Jolliffe T, Baron-Cohen S. Are people with autism and Asperger syndrome faster than normal on the Embedded Figures Test? J Child Psychol Psychiatry 1997;38:527-534.

38) Witkin HA. A manual for the embedded figures tests. Palo Alto, CA: Consulting Psychologists Press; 1971.

39) Wechsler D. WAIS-R Manual: Wechsler Adult Intelligence Scalesrevised. New York, NY: Psychological Corporation;1981.

40) Kana RK, Liu Y, Williams DL, Keller TA, Schipul SE, Minshew NJ, et al. The local, global, and neural aspects of visuospatial processing in autism spectrum disorders. Neuropsychologia 2013;51: 2995-3003.

41) Keehn B, Brenner L, Palmer E, Lincoln AJ, Müller RA. Functional brain organization for visual search in ASD. J Int Neuropsychol Soc 2008;14:990-1003.

42) Ring HA, Baron-Cohen S, Wheelwright S, Williams SC, Brammer M, Andrew C, et al. Cerebral correlates of preserved cognitive skills in autism: a functional MRI study of embedded figures task performance. Brain 1999;122:1305-1315.

43) Kosslyn SM, Thompson WL, Kim IJ, Alpert NM. Topographical representations of mental images in primary visual cortex. Nature 1995;378:496-498.

44) Manjaly ZM, Bruning N, Neufang S, Stephan KE, Brieber S, Marshall JC, et al. Neurophysiological correlates of relatively enhanced local visual search in autistic adolescents. Neuroimage 2007;35: 283-291.

45) Damarla SR, Keller TA, Kana RK, Cherkassky VL, Williams DL, Minshew NJ, et al. Cortical underconnectivity coupled with preserved visuospatial cognition in autism: evidence from an fMRI study of an embedded figures task. Autism Res 2010;3:273-279.

46) Lee PS, Foss-Feig J, Henderson JG, Kenworthy LE, Gilotty L, Gaillard WD, et al. Atypical neural substrates of embedded figures task performance in children with autism spectrum disorder. Neuroimage 2007;38:184-193.

47) Wager TD, Smith EE. Neuroimaging studies of working memory: a meta-analysis. Cogn Affect Behav Neurosci 2003;3:255-274.

48) Spencer MD, Holt RJ, Chura LR, Calder AJ, Suckling J, Bullmore ET, et al. Atypical activation during the embedded figures task as a functional magnetic resonance imaging endophenotype of autism. Brain 2012;135:3469-3480.

49) Keehn B, Shih P, Brenner LA, Townsend J, Müller RA. Functional connectivity for an "island of sparing" in autism spectrum disorder: an fMRI study of visual search. Hum Brain Mapp 2013;34: 2524-2537.

50) Happé F, Frith U. The weak coherence account: detail-focused cognitive style in autism spectrum disorders. J Autism Dev Disord 2006;36:5-25.

51) Critchley HD, Daly EM, Bullmore ET, Williams SC, Van Amelsvoort $T$, Robertson DM, et al. The functional neuroanatomy of 
social behaviour: changes in cerebral blood flow when people with autistic disorder process facial expressions. Brain 2000;123: 2203-2212.

52) Belmonte MK, Yurgelun-Todd DA. Functional anatomy of impaired selective attention and compensatory processing in autism. Brain Res Cogn Brain Res 2003;17:651-664.

53) Just MA, Cherkassky VL, Keller TA, Minshew NJ. Cortical activation and synchronization during sentence comprehension in high-functioning autism: evidence of underconnectivity. Brain 2004;127:1811-1821.

54) Jones TB, Bandettini PA, Kenworthy L, Case LK, Milleville SC, Martin A, et al. Sources of group differences in functional connectivity: an investigation applied to autism spectrum disorder. Neuroimage 2010;49:401-414.

55) Samson F, Mottron L, Soulières I, Zeffiro TA. Enhanced visual functioning in autism: an ALE meta-analysis. Hum Brain Mapp 2012;33:1553-1581.

56) Wang S, Jiang M, Duchesne XM, Laugeson EA, Kennedy DP, Adolphs R, et al. Atypical visual saliency in autism spectrum disorder quantified through model-based eye tracking. Neuron 2015; 88:604-616.

57) Caron MJ, Mottron L, Berthiaume C, Dawson M. Cognitive mechanisms, specificity and neural underpinnings of visuospatial peaks in autism. Brain 2006;129:1789-1802.

58) Lord C, Risi S, Lambrecht L, Cook EH Jr, Leventhal BL, DiLavore PC, et al. The Autism Diagnostic Observation Schedule-Generic: a standard measure of social and communication deficits associated with the spectrum of autism. J Autism Dev Disord 2000; 30:205-223.

59) Stark CE, Squire LR. When zero is not zero: the problem of ambiguous baseline conditions in fMRI. Proc Natl Acad Sci U S A 2001;98:12760-12766.

60) Bertone A, Mottron L, Jelenic P, Faubert J. Motion perception in autism: a "complex" issue. J Cogn Neurosci 2003;15:218-225.

61) Pellicano E, Gibson L, Maybery M, Durkin K, Badcock DR. Abnormal global processing along the dorsal visual pathway in autism: a possible mechanism for weak visuospatial coherence? Neuropsychologia 2005;43:1044-1053.

62) Cavanagh P. Short-range vs long-range motion: not a valid distinction. Spat Vis 1991;5:303-309.

63) Kaiser MD, Shiffrar M. The visual perception of motion by observers with autism spectrum disorders: a review and synthesis. Psychon Bull Rev 2009;16:761-777.

64) Koldewyn K, Whitney D, Rivera SM. The psychophysics of visual motion and global form processing in autism. Brain 2010;133: 599-610.

65) Robertson CE, Martin A, Baker CI, Baron-Cohen S. Atypical integration of motion signals in autism spectrum conditions. PLoS One 2012;7:e48173.

66) Milner AD, Goodale MA. Two visual systems re-viewed. Neuropsychologia 2008;46:774-785.

67) Tobimatsu S, Celesia GG. Studies of human visual pathophysiology with visual evoked potentials. Clin Neurophysiol 2006;117: 1414-1433.

68) Rees G, Friston K, Koch C. A direct quantitative relationship between the functional properties of human and macaque V5. Nat Neurosci 2000;3:716-723.

69) Macintyre-Beon C, Ibrahim H, Hay I, Cockburn D, Calvert J, Bowman R. Dorsal stream dysfunction in children. A review and an approach to diagnosis and management. Curr Pediatr Rev 2010;6:166-182.

70) Williams JH, Whiten A, Singh T. A systematic review of action imitation in autistic spectrum disorder. J Autism Dev Disord 2004; 34:285-299.

71) Mottron L, Mineau S, Martel G, Bernier CS, Berthiaume C,
Dawson M, et al. Lateral glances toward moving stimuli among young children with autism: early regulation of locally oriented perception? Dev Psychopathol 2007;19:23-36.

72) Brieber S, Herpertz-Dahlmann B, Fink GR, Kamp-Becker I, Remschmidt H, Konrad K. Coherent motion processing in autism spectrum disorder (ASD): an fMRI study. Neuropsychologia 2010;48: 1644-1651.

73) Koldewyn K, Whitney D, Rivera SM. Neural correlates of coherent and biological motion perception in autism. Dev Sci 2011;14: 1075-1088.

74) Robertson CE, Thomas C, Kravitz DJ, Wallace GL, Baron-Cohen S, Martin A, et al. Global motion perception deficits in autism are reflected as early as primary visual cortex. Brain 2014;137:25882599.

75) Takarae Y, Luna B, Minshew NJ, Sweeney JA. Visual motion processing and visual sensorimotor control in autism. J Int Neuropsychol Soc 2014;20:113-122.

76) Peiker I, Schneider TR, Milne E, Schöttle D, Vogeley K, Münchau A, et al. Stronger neural modulation by visual motion intensity in autism spectrum disorders. PLoS One 2015;10:e0132531.

77) Cardin JA, Carlén M, Meletis K, Knoblich U, Zhang F, Deisseroth $\mathbf{K}$, et al. Driving fast-spiking cells induces gamma rhythm and controls sensory responses. Nature 2009;459:663-667.

78) Donner TH, Siegel M. A framework for local cortical oscillation patterns. Trends Cogn Sci 2011:15:191-199.

79) Siegel M, Donner TH, Oostenveld R, Fries P, Engel AK. Highfrequency activity in human visual cortex is modulated by visual motion strength. Cereb Cortex 2007;17:732-741.

80) Robertson CE, Ratai EM, Kanwisher N. Reduced GABAergic action in the autistic brain. Curr Biol 2016;26:80-85.

81) Vandenbroucke MW, Scholte HS, van Engeland H, Lamme VA, Kemner C. A neural substrate for atypical low-level visual processing in autism spectrum disorder. Brain 2008;131:1013-1024.

82) Sanchez-Marin FJ, Padilla-Medina JA. A psychophysical test of the visual pathway of children with autism. J Autism Dev Disord 2008;38:1270-1277.

83) Milne E. Increased intra-participant variability in children with autistic spectrum disorders: evidence from single-trial analysis of evoked EEG. Front Psychol 2011;2:51

84) Simion F, Regolin L, Bulf $H$. A predisposition for biological motion in the newborn baby. Proc Natl Acad Sci U S A 2008;105:809-813.

85) Fox R, McDaniel C. The perception of biological motion by human infants. Science 1982;218:486-487.

86) Pavlova MA. Biological motion processing as a hallmark of social cognition. Cereb Cortex 2012;22:981-995.

87) Klin A, Lin DJ, Gorrindo P, Ramsay G, Jones W. Two-year-olds with autism orient to non-social contingencies rather than biological motion. Nature 2009;459:257-261.

88) Kaiser MD, Pelphrey KA. Disrupted action perception in autism: behavioral evidence, neuroendophenotypes, and diagnostic utility. Dev Cogn Neurosci 2012;2:25-35.

89) Hubert B, Wicker B, Moore DG, Monfardini E, Duverger H, Da Fonséca D, et al. Brief report: recognition of emotional and nonemotional biological motion in individuals with autistic spectrum disorders. J Autism Dev Disord 2007;37:1386-1392.

90) Nackaerts E, Wagemans J, Helsen W, Swinnen SP, Wenderoth N, Alaerts K. Recognizing biological motion and emotions from point-light displays in autism spectrum disorders. PLoS One 2012;7:e44473.

91) Lahnakoski JM, Glerean E, Salmi J, Jääskeläinen IP, Sams M, Hari R, et al. Naturalistic fMRI mapping reveals superior temporal sulcus as the hub for the distributed brain network for social perception. Front Hum Neurosci 2012;6:233.

92) Kana RK, Libero LE, Hu CP, Deshpande HD, Colburn JS. Functional brain networks and white matter underlying theory-of-mind 
in autism. Soc Cogn Affect Neurosci 2014;9:98-105.

93) Pierce K, Haist F, Sedaghat F, Courchesne E. The brain response to personally familiar faces in autism: findings of fusiform activity and beyond. Brain 2004;127:2703-2716.

94) Boddaert N, Zilbovicius M. Functional neuroimaging and childhood autism. Pediatr Radiol 2002;32:1-7.

95) Herrington JD, Baron-Cohen S, Wheelwright SJ, Singh KD, Bullmore ET, Brammer M, et al. The role of MT+/V5 during biological motion perception in Asperger syndrome: an fMRI study. Res Autism Spectr Disord 2007;1:14-27.

96) Freitag CM, Konrad C, Häberlen M, Kleser C, von Gontard A, Reith W, et al. Perception of biological motion in autism spectrum disorders. Neuropsychologia 2008;46:1480-1494.

97) Kaiser MD, Hudac CM, Shultz S, Lee SM, Cheung C, Berken AM, et al. Neural signatures of autism. Proc Natl Acad Sci U S A 2010; 107:21223-21228.

98) Manning C, Tibber MS, Charman T, Dakin SC, Pellicano E. Enhanced integration of motion information in children with autism. J Neurosci 2015;35:6979-6986.

99) Alaerts K, Swinnen SP, Wenderoth N. Neural processing of biological motion in autism: an investigation of brain activity and effective connectivity. Sci Rep 2017;7:5612.

100) Jack A, Keifer CM, Pelphrey KA. Cerebellar contributions to biological motion perception in autism and typical development. Hum Brain Mapp 2017;38:1914-1932.

101) Ramnani N. The primate cortico-cerebellar system: anatomy and function. Nat Rev Neurosci 2006;7:511-522.

102) Park WJ, Schauder KB, Zhang R, Bennetto L, Tadin D. High internal noise and poor external noise filtering characterize perception in autism spectrum disorder. Sci Rep 2017;7:17584.

103) Nomi JS, Uddin LQ. Face processing in autism spectrum disorders: from brain regions to brain networks. Neuropsychologia 2015; 71:201-216.

104) Scherf KS, Behrmann M, Humphreys K, Luna B. Visual categoryselectivity for faces, places and objects emerges along different developmental trajectories. Dev Sci 2007;10:F15-F30.

105) Behrmann M, Avidan G, Leonard GL, Kimchi R, Luna B, Humphreys $\mathrm{K}$, et al. Configural processing in autism and its relationship to face processing. Neuropsychologia 2006;44:110-129.

106) Gross TF. The perception of four basic emotions in human and nonhuman faces by children with autism and other developmental disabilities. J Abnorm Child Psychol 2004;32:469-480.

107) Bar-Haim Y, Shulman C, Lamy D, Reuveni A. Attention to eyes and mouth in high-functioning children with autism. J Autism Dev Disord 2006;36:131-137.

108) Haxby JV, Hoffman EA, Gobbini MI. The distributed human neural system for face perception. Trends Cogn Sci 2000;4:223-233.

109) Liu J, Harris A, Kanwisher N. Stages of processing in face perception: an MEG study. Nat Neurosci 2002;5:910-916.

110) Harris A, Aguirre GK. Neural tuning for face wholes and parts in human fusiform gyrus revealed by FMRI adaptation. J Neurophysiol 2010;104:336-345.

111) Sato $W$, Toichi $M$, Uono $S$, Kochiyama T. Impaired social brain network for processing dynamic facial expressions in autism spectrum disorders. BMC Neurosci 2012;13:99.

112) Wang S, Tudusciuc O, Mamelak AN, Ross IB, Adolphs R, Rutishauser $\mathrm{U}$. Neurons in the human amygdala selective for perceived emotion. Proc Natl Acad Sci U S A 2014;111:E3110-E3119.

113) Humphreys K, Hasson U, Avidan G, Minshew N, Behrmann M. Cortical patterns of category-selective activation for faces, places and objects in adults with autism. Autism Res 2008;1:52-63.

114) Bookheimer SY, Wang AT, Scott A, Sigman M, Dapretto M. Frontal contributions to face processing differences in autism: evidence from fMRI of inverted face processing. J Int Neuropsychol Soc 2008;14:922-932.
115) Kleinhans NM, Johnson LC, Richards T, Mahurin R, Greenson J, Dawson G, et al. Reduced neural habituation in the amygdala and social impairments in autism spectrum disorders. Am J Psychiatry 2009;166:467-475.

116) Domes G, Heinrichs M, Kumbier E, Grossmann A, Hauenstein K, Herpertz SC. Effects of intranasal oxytocin on the neural basis of face processing in autism spectrum disorder. Biol Psychiatry 2013; 74:164-171.

117) Pierce K, Redcay E. Fusiform function in children with an autism spectrum disorder is a matter of "who." Biol Psychiatry 2008;64: 552-560.

118) Tottenham N, Hertzig ME, Gillespie-Lynch K, Gilhooly T, Millner AJ, Casey BJ. Elevated amygdala response to faces and gaze aversion in autism spectrum disorder. Soc Cogn Affect Neurosci 2014;9:106-117.

119) Weng SJ, Carrasco M, Swartz JR, Wiggins JL, Kurapati N, Liberzon I, et al. Neural activation to emotional faces in adolescents with autism spectrum disorders. J Child Psychol Psychiatry 2011; 52:296-305

120) Kylliäinen A, Hietanen JK. Skin conductance responses to another person's gaze in children with autism. J Autism Dev Disord 2006;36:517-525.

121) Ashwin C, Baron-Cohen S, Wheelwright S, O'Riordan M, Bullmore ET. Differential activation of the amygdala and the 'social brain' during fearful face-processing in Asperger Syndrome. Neuropsychologia 2007;45:2-14.

122) Malisza KL, Clancy C, Shiloff D, Holden J, Jones C, Paulson K, et al. Functional magnetic resonance imaging of facial information processing in children with autistic disorder, attention deficit hyperactivity disorder and typically developing controls. Int J Adolesc Med Health 2011;23:269-277.

123) Dapretto M, Davies MS, Pfeifer JH, Scott AA, Sigman M, Bookheimer SY, et al. Understanding emotions in others: mirror neuron dysfunction in children with autism spectrum disorders. Nat Neurosci 2006;9:28-30.

124) Pitcher D, Dilks DD, Saxe RR, Triantafyllou C, Kanwisher N. Differential selectivity for dynamic versus static information in face-selective cortical regions. Neuroimage 2011;56:2356-2363.

125) Bernstein M, Yovel G. Two neural pathways of face processing: a critical evaluation of current models. Neurosci Biobehav Rev 2015; 55:536-546.

126) Bernstein M, Erez Y, Blank I, Yovel G. An integrated neural framework for dynamic and static face processing. Sci Rep 2018;8:7036.

127) Pelphrey KA, Morris JP, McCarthy G, Labar KS. Perception of dynamic changes in facial affect and identity in autism. Soc Cogn Affect Neurosci 2007;2:140-149.

128) Borowiak K, Maguinness C, von Kriegstein K. Dorsal-movement and ventral-form regions are functionally connected during visual-speech recognition. Hum Brain Mapp 2020;41:952-972.

129) Kliemann D, Richardson H, Anzellotti S, Ayyash D, Haskins AJ, Gabrieli JDE, et al. Cortical responses to dynamic emotional facial expressions generalize across stimuli, and are sensitive to taskrelevance, in adults with and without Autism. Cortex 2018;103: 24-43.

130) Johnson MH. Subcortical face processing. Nat Rev Neurosci 2005; 6:766-774

131) Vuilleumier P, Armony JL, Driver J, Dolan RJ. Distinct spatial frequency sensitivities for processing faces and emotional expressions. Nat Neurosci 2003;6:624-631.

132) Osterling JA, Dawson G, Munson JA. Early recognition of 1-yearold infants with autism spectrum disorder versus mental retardation. Dev Psychopathol 2002;14:239-251.

133) Dawson G, Webb SJ, McPartland J. Understanding the nature of face processing impairment in autism: insights from behavioral and electrophysiological studies. Dev Neuropsychol 2005;27:403- 
424.

134) Celeghin A, Galetto V, Tamietto M, Zettin M. Emotion recognition in low-spatial frequencies is partly preserved following traumatic brain injury. Biomed Res Int 2019;2019:9562935.

135) Vlamings PH, Jonkman LM, van Daalen E, van der Gaag RJ, Kemner C. Basic abnormalities in visual processing affect face processing at an early age in autism spectrum disorder. Biol Psychiatry 2010;68:1107-1113.

136) Corradi-Dell'acqua C, Schwartz S, Meaux E, Hubert B, Vuilleumier P, Deruelle C. Neural responses to emotional expression information in high- and low-spatial frequency in autism: evidence for a cortical dysfunction. Front Hum Neurosci 2014;8:189.

137) Ionescu MR. Subliminal perception of complex visual stimuli. Rom J Ophthalmol 2016;60:226-230.

138) Brooks SJ, Savov V, Allzén E, Benedict C, Fredriksson R, Schiöth HB. Exposure to subliminal arousing stimuli induces robust activation in the amygdala, hippocampus, anterior cingulate, insular cortex and primary visual cortex: a systematic meta-analysis of fMRI studies. Neuroimage 2012;59:2962-2973.

139) Kleinhans NM, Richards T, Johnson LC, Weaver KE, Greenson J, Dawson G, et al. fMRI evidence of neural abnormalities in the subcortical face processing system in ASD. Neuroimage 2011;54: 697-704.

140) Hall GB, Doyle KA, Goldberg J, West D, Szatmari P. Amygdala engagement in response to subthreshold presentations of anxious face stimuli in adults with autism spectrum disorders: preliminary insights. PLoS One 2010;5:e10804.

141) Hadjikhani N, Åsberg Johnels J, Zürcher NR, Lassalle A, Guillon $\mathrm{Q}$, Hippolyte L, et al. Look me in the eyes: constraining gaze in the eye-region provokes abnormally high subcortical activation in autism. Sci Rep 2017;7:3163.

142) Elsabbagh M, Johnson MH. Getting answers from babies about autism. Trends Cogn Sci 2010;14:81-87. 